\title{
Soluble Intracellular Adhesion Molecule-1 in Patients with Unipolar or Bipolar Affective Disorders: Results from a Pilot Trial
}

\author{
Martin Schaefer ${ }^{a, b}$ Susanne Sarkar ${ }^{b, c}$ Markus Schwarz ${ }^{d}$ Astrid Friebe $^{e}$ \\ a Department of Psychiatry, Psychotherapy, Psychosomatics and Addiction Medicine, Kliniken Essen-Mitte, Essen, \\ ${ }^{b}$ Department of Psychiatry and Psychotherapy, Charité - Universitätsmedizin Berlin, Charité Campus Mitte, Berlin, \\ 'Department and Outpatient Clinic of Medical Psychology, Center of Psychosocial Medicine, University Medical \\ Center Hamburg-Eppendorf, Hamburg, d Institut für Laboratoriumsmedizin, Ludwig Maximilian University, Munich, \\ and ${ }^{e}$ Department of Psychiatry, LWL University Hospital, Ruhr University Bochum, Bochum, Germany
}

\section{Key Words}

Bipolar disorder · Depression · Soluble intracellular adhesion molecule-1 - Cell adhesion molecules · Mood changes - Mania

\begin{abstract}
Background: Immunological and vascular markers may play a role in the pathophysiology of mood disorders and mood changes. Aim: To test whether the cell adhesion molecule soluble intracellular adhesion molecule-1 (sICAM-1) may serve as a biomarker for patients with unipolar or bipolar affective disorders when compared to a healthy control group, and whether sICAM-1 blood levels change during different mood states. Methods: sICAM-1 serum concentrations were compared between 20 healthy controls and 48 patients with affective disorders (unipolar, bipolar II and bipolar I disorder) during different mood states (euthymic mood state, depression or mania). Results: When compared to healthy controls, patients with affective disorders had significantly higher sICAM-1 levels during the euthymic state $(p=0.015)$. Differences became more pronounced during depression $(p=$ 0.013). When unipolar and bipolar patients were analyzed separately, unipolar patients significantly differed from con-
\end{abstract}

\section{KARGER}

(c) 2016 S. Karger AG, Basel

E-Mail karger@karger.com

www.karger.com/nps trols during the euthymic and depressive mood state, while bipolar II patients showed a trend towards higher sICAM-1 levels during depression. Patients with bipolar I disorders had significantly higher sICAM-1 levels during manic states when compared to controls ( $p=0.007)$. Conclusions: sICAM-1 elevation in unipolar and bipolar patients, independent of mood changes, might support the hypothesis of chronic immune activation and endothelial dysfunction in patients with affective disorders.

(c) 2016 S. Karger AG, Basel

\section{Introduction}

Recent findings suggest that chronic immune stimulation, including higher levels of pro-inflammatory cytokines, acute-phase proteins, chemokines and cellular adhesion molecules, may play a role in the pathophysiology of mood changes in unipolar and bipolar disorders $[1,2]$. The most consistent findings are elevated interleukin-6 (IL-6) and tumor necrosis factor alpha (TNF- $\alpha$ ) levels in patients with unipolar depressive disorders [3] and an increase in soluble IL-2 receptor (sIL-2R), TNF- $\alpha$, soluble tumor necrosis factor receptor type 1 (sTNFR1), sIL-6R 
and IL-4 in patients with bipolar disorder [1]. However, it remains unclear how peripheral immune activation might affect the brain and induce mood changes like depression or mania if the blood-brain barrier $(\mathrm{BBB})$ is intact.

The intracellular adhesion molecule-1 (ICAM-1) on the $\mathrm{BBB}$ endothelial cells appears to be a major mediator of leukocyte immigration into the central nervous system. Soluble ICAM-1 (sICAM-1) is an indirect parameter of increased ICAM-1 expression on activated immune or endothelial cells. In inflammatory neurological diseases, elevated levels of soluble ICAM-1 are assumed to be an indicator for an impairment of the $\mathrm{BBB}$, with an increased invasion of macrophages/microglia or cytokines in the central nervous system [4-6]. Elevated blood levels of the cell adhesion molecule ICAM-1 have been shown to be associated with depressive mood states in elderly patients, patients with vascular-related depression, or patients with depression during immune treatment [7-10]. We recently reported a correlation between elevated plasma levels of sICAM-1 and depressive symptoms in patients with malignant melanoma who received adjuvant therapy with the cytokine interferon-alpha [9]. Elevations in sICAM-1 have further been observed in patients with chronic stress as a risk factor for both immune activation and depressive mood changes [11]. Finally, higher sICAM-1 levels in the cerebrospinal fluid were very recently shown to be predictive for depression in individuals with traumatic brain injuries [12]. Taken together, ICAM-1 might be involved in the pathophysiology of vascular- and immune-associated mood changes. We therefore investigated in a pilot trial sICAM-1 concentrations in patients with unipolar or bipolar affective disorders during different mood states (euthymia, depression or mania) and compared them to those of healthy controls. Our trial aimed to explore different or abnormal adhesion molecule expression (sICAM-1) in unipolar and bipolar mood disorders, to compare this to healthy controls, and to determine its possible role as a biomarker.

\section{Patients and Methods}

Patients were eligible for the study if they were between 18 and 70 years old and had a diagnosis of unipolar depression or bipolar disorder according to the diagnostic criteria of DSM-IV and ICD10. Exclusion criteria were any other axis I disorders (DSM-IV), significant chronically immunological or acute inflammatory disorders, or severe neurological, cardiovascular, hepatic, renal, metabolic, or other severe somatic diseases. We also excluded patients with clinically relevant abnormalities in laboratory tests and fe- male subjects who are pregnant or breastfeeding. Participants were grouped into different diagnostic categories: patients with unipolar or bipolar affective disorder (bipolar II and bipolar I) using the Structured Clinical Interview for DSM-IV axis I disorders (SCID). Data from patients with affective disorders was compared with those of a control group matched for age and gender without any history of psychiatric disorders. Controls were also free of somatic disorders, acute infections or chronic immune disorders and did not receive medication. The study was approved by the local ethics review board. All patients gave their informed consent.

\section{Clinical Ratings}

A diagnosis of acute depressive or manic episodes was made by an experienced psychiatrist according to the DSM-IV criteria. The severity of depression or mania was evaluated with the Hamilton Depression Scale (HAMD, 17-item version) and with the Young Mania Rating Scale (YMRS). Patients were not included during hypomanic episodes. Euthymic patients did not show manic or depressive symptoms and had to have been stable for the last 4 weeks. All patients were monitored over a period of 12 months, and if possible, blood was taken during different mood states. If patients were included during a euthymic state, they were followed for up to 12 months in our outpatient clinic and seen every 4 weeks to detect possible relevant depressive or manic episodes. Whenever possible, patients who were included during a period of depression or mania were followed after treatment to collect data during stabilized euthymic episodes.

\section{Measurements}

sICAM-1 was measured during different mood states (euthymic mood state or acute depression). Overall, we compared 20 controls with 48 patients who had affective disorders: 11 patients with unipolar depression, 13 patients with bipolar II disorder (BPII) and 24 patients with bipolar I disorder (BP-I). For the evaluation of sICAM-1, blood was taken between 9:00 and 12:00 a.m. Serum levels of sICAM-1 were measured by a commercially available double-sandwich ELISA (CELLFREE human sICAM-1, Endogen, Rockford, Ill., USA). The detection limit was $0.3 \mathrm{ng} / \mathrm{ml}$. The mean values for intra- and inter-assay variability were found to be $<7.5 \%$. Laboratory personnel were unaware of the patient's diagnosis or clinical status. All measurements were done in duplicates. The paired samples of one patient were measured within one assay. All assays of the immune parameters were carried out with a single lot number of reagents and consumables employed by a single operator.

\section{Statistical Evaluation}

For the systematic evaluation of sICAM-1, a stepwise comparison of patients and healthy controls was performed with independent $t$ tests. The stepwise data analysis was also used because of the pilot character of the trial and the relatively low number of patients in the subgroups. In the first step, sICAM-1 levels were compared between patients and controls in general. In the second step, we used univariate analysis of variance (ANOVA) to compare sICAM-1 levels between patients with unipolar affective disorders, bipolar affective disorders and controls. In the third step, we were interested in detecting the differences between patients in the BP-I and BP-II subgroups, unipolar patients and controls. Linear regression analysis was used to analyze the influence of possible confounders including age, gender and medication (antidepressants, 
Table 1. Baseline data

\begin{tabular}{|c|c|c|c|c|}
\hline & $\begin{array}{l}\text { Controls } \\
(\mathrm{n}=20)\end{array}$ & $\begin{array}{l}\text { Unipolar } \\
(\mathrm{n}=11)\end{array}$ & $\begin{array}{l}\text { BP-II } \\
(n=13)\end{array}$ & $\begin{array}{l}\text { BP-I } \\
(\mathrm{n}=24)\end{array}$ \\
\hline Age, years & $46.1 \pm 13.9$ & $55.8 \pm 12.2$ & $49.1 \pm 17.8$ & $38.67 \pm 15.2$ \\
\hline Female & $13(65 \%)$ & $8(73 \%)$ & $11(85 \%)$ & $13(54 \%)$ \\
\hline \multicolumn{5}{|l|}{ Medication } \\
\hline No medication & $20(100 \%)$ & $2(18 \%)$ & 0 & 0 \\
\hline $\mathrm{AD}$ & 0 & $5(45 \%)$ & $7(54 \%)$ & 0 \\
\hline MS & 0 & 0 & $1(8 \%)$ & $9(38 \%)$ \\
\hline $\mathrm{AP}$ & 0 & $1(9 \%)$ & 0 & $1(4 \%)$ \\
\hline $\mathrm{BD}$ & 0 & 0 & 0 & $1(4 \%)$ \\
\hline $\mathrm{AD}+\mathrm{MS}$ & 0 & $1(9 \%)$ & $2(15 \%)$ & $2(8 \%)$ \\
\hline $\mathrm{AD}+\mathrm{AP}$ & 0 & $1(9 \%)$ & $2(15 \%)$ & $6(25 \%)$ \\
\hline $\mathrm{MS}+\mathrm{AP}$ & 0 & 0 & 0 & $3(13 \%)$ \\
\hline $\mathrm{AD}+\mathrm{MS}+\mathrm{AP}$ & 0 & $1(9 \%)$ & 0 & $2(8 \%)$ \\
\hline \multicolumn{5}{|l|}{ HAMD } \\
\hline Euthymic & & $8.3 \pm 2.9(n=10)$ & $7.4 \pm 6.9(\mathrm{n}=12)$ & $3.6 \pm 3.9(n=23)$ \\
\hline Depressive & & $28.6 \pm 5.4(\mathrm{n}=11)$ & $25.8 \pm 7.8(\mathrm{n}=13)$ & $24.2 \pm 15.1(\mathrm{n}=9)$ \\
\hline \multicolumn{5}{|l|}{ YMRS } \\
\hline Euthymic & & $1.4 \pm 1.7(n=10)$ & $1.4 \pm 1.1(\mathrm{n}=12)$ & $4.3 \pm 3.8(n=23)$ \\
\hline Depressive & & $2.4 \pm 1.3(\mathrm{n}=11)$ & $2.6 \pm 1.5(\mathrm{n}=13)$ & $3.0 \pm 2.5(\mathrm{n}=9)$ \\
\hline
\end{tabular}

mood stabilizers, antipsychotics or combination therapy) concerning sICAM-1 levels in unipolar and bipolar patients during euthymic, depressive or manic mood states. Finally, we compared sICAM-1 levels between different mood states independent of diagnosis by t tests (for two groups) or ANOVA (for more than two groups). For baseline analysis, we compared continuous variables with independent $t$ tests and categorical variables with $\chi^{2}$ tests. The association between depression and mania ratings scores on the one hand and sICAM-1 serum levels on the other was analyzed by using Pearson's correlation. Bonferroni adjustment was used to correct for multiple comparisons.

\section{Results}

Overall data of 20 healthy controls was compared with those of 48 patients: 11 with unipolar and 37 with bipolar affective disorder (24 BP-I and 13 BP-II). The baseline data are shown in table 1 . Significant differences between the unipolar, BP-I and BP-II patients during euthymic mood states was found for HAMD $\left(\mathrm{F}_{2,42}=4.515, \mathrm{p}=\right.$ $0.017)$ and YMRS $\left(\mathrm{F}_{2,42}=5.505, \mathrm{p}=0.008\right)$. During euthymic mood states, unipolar patients had significantly higher HAMD scores when compared to BP-I patients $(\mathrm{p}=0.038)$. BP-I patients showed significantly higher scores in the YMRS when compared to unipolar ( $\mathrm{p}=$ $0.012)$ or BP-II patients $(\mathrm{p}=0.008)$. Based on the trial's naturalistic design, the type and dosage of medication was not restricted. Medication had to have been stable for at least 1 week. Most patients were treated with mood stabilizers (lithium, valproate) and/or antidepressants (venlafaxine, sertraline, paroxetine, amitriptyline) and/or atypical antipsychotics (olanzapine, risperidone). Only 4 patients received typical antipsychotics (perazine, haloperidol, flupentixol).

\section{sICAM-1 Concentrations Differ between Euthymic Patients and Controls}

Stable euthymic patients with unipolar or bipolar mood disorders had significantly higher sICAM-1 concentrations when compared to healthy controls $(t=2.501$, d.f. $=$ $58, \mathrm{p}=0.015$ ) (fig. 1a). A comparison of sICAM-1 levels (ANOVA) between unipolar, BP-I and BP-II patients and healthy controls confirmed a significant difference between the subgroup of unipolar patients and healthy controls ( $\mathrm{p}=0.018,95 \%$ CI 18.5-187.3) (fig. 1b). However, BP-II patients showed a strong trend towards higher sICAM-1 levels compared to controls ( $\mathrm{p}=0.057,95 \%$ CI -2.3 to 160.5$)$. sICAM-1 levels did not differ between pa- 
Fig. 1. a Comparison of sICAM-1 levels in stable euthymic, depressive or manic patients with unipolar or bipolar mood disorders and healthy controls (mean $\pm \mathrm{SD}$ ). b Comparison of sICAM-1 levels (ANOVA) between healthy controls and unipolar, BP-II and BP-I patients during the euthymic, depressive or manic state.
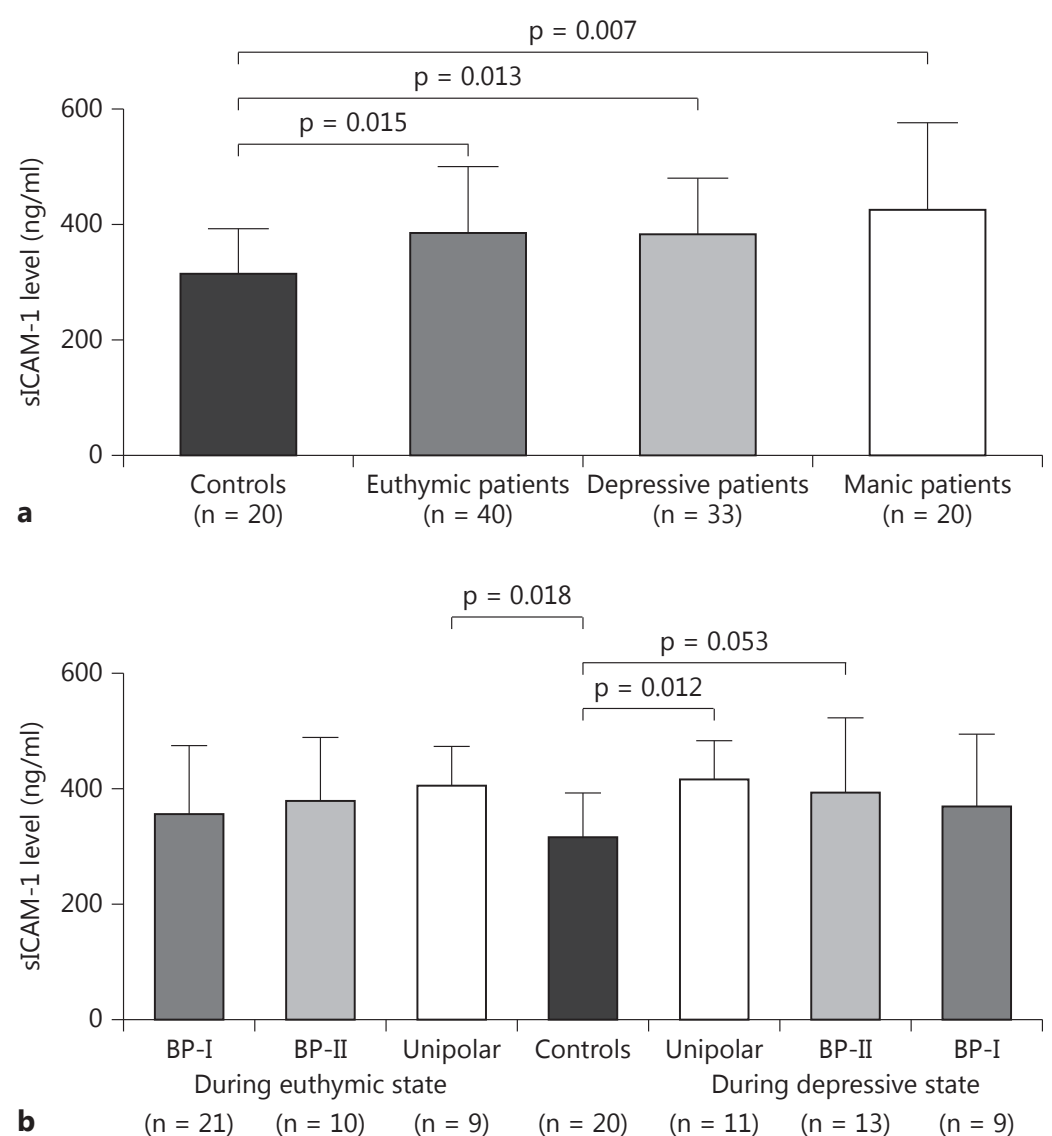

b $(n=20)$ $(n=9)$ tients with unipolar or bipolar disorder. Concentrations of sICAM-1 did not correlate with depression scores ( $\mathrm{r}=$ $-0.207, p=0.299)$, but were positively correlated with age $(\mathrm{r}=0.345, \mathrm{p}=0.029)$. According to regression analysis, age, gender and medication were not associated with sICAM-1 levels in unipolar or bipolar patients.

sICAM-1 Levels Are Increased during Depression and Mania when Compared to Healthy Controls

During depression, patients with mood disorders showed significantly higher sICAM-1 concentrations when compared to controls $(t=2.584$, d.f. $=51, \mathrm{p}=0.013$ ) (fig. 1a). Regarding the subgroups, sICAM-1 levels were significantly higher in unipolar patients when compared to controls ( $\mathrm{p}=0.012,95 \%$ CI 21.2-160.5). BP-II patients showed a strong trend towards higher sICAM-1 levels compared to controls ( $\mathrm{p}=0.053,95 \% \mathrm{CI}-0.87$ to 131.3 ) (fig. 1b). sICAM-1 levels did not differ between unipolar and bipolar patients during depression $(\mathrm{p}=0.301,95 \%$

sICAM-1 in Patients with Affective Disorders
CI -32.64 to 103.43 ), and sICAM-1 levels were not correlated with the severity of depression (HAMD score).

In patients with BP-I disorder, sICAM-1 concentrations were significantly higher during mania as compared to controls $(\mathrm{t}=2.871$, d.f. $=33, \mathrm{p}=0.007)$ (fig. 1a). sICAM-1 levels were again not correlated with symptom severity (YMRS, $r=-0.132, p=0.639$ ), but positively correlated with age $(\mathrm{r}=0.524, \mathrm{p}=0.045)$. Other possible confounders such as gender and medication were not significantly related to sICAM-1 concentrations in unipolar and bipolar patients.

sICAM-1 Levels Did Not Show Changes in a Subgroup of Patients with Remission after Mania or Depression

We also looked at intra-individual changes of sICAM1 blood levels in the subgroup of patients with unipolar and bipolar disorders who could be followed during different mood states (euthymia versus depression or mania). sICAM-1 levels did not differ in unipolar or BP-II 
patients who recovered and reached a euthymic mood state when compared to depression (unipolar patients: $\mathrm{F}_{1,8}=0.312, \mathrm{p}=0.592$; BP-II patients: $\mathrm{F}_{1,9}=0.013, \mathrm{p}=$ 0.912 ). In addition, sICAM-1 levels did not differ in remitted BP-I patients with a euthymic mood state compared to blood levels during their manic episode $(\mathrm{t}=$ 0.818 , d.f. $=12, \mathrm{p}=0.429)$ or depression $(\mathrm{t}=1.167$, d.f. $=$ $1, \mathrm{p}=0.451)$.

\section{Discussion}

Our findings demonstrate for the first time, independent of mood changes, a stable sICAM-1 elevation in patients with unipolar and bipolar affective disorders when compared to a healthy control group. However, when subgroups in our trial were compared separately, differences only became significant for patients with unipolar affective disorders, based on the relatively low number of patients per group. A further notable observation were the mild but continuous differences between unipolar, BP-II and BP-I patients, possibly indicating a discriminating property of sICAM-1 between unipolar and bipolar disorders and supporting the concept of diagnostic differentiation between unipolar, BP-II and BP-I patients. However, differences between the patient subgroups (unipolar, BP-I and BP-II) were not significant and have to be reinvestigated with larger collectives. sICAM-1 levels did not differ between patients with acute episodes of depression or mania when compared to patients in a stable euthymic state. This observation may support the hypothesis of a trait marker for patients with unipolar or bipolar affective disorders.

sICAM-1 has been used in clinical trials as a biomarker for endothelial activation and dysfunction [13]. It has also been investigated as an important marker in a vascular disease animal model of depression [14]. Endothelial dysfunction contributes to the pathogenesis, severity and progression of a variety of potentially serious infectious diseases and syndromes [15]. In addition, increased sICAM-1 concentrations have been found before or during neuroinflammatory conditions such as tick-borne encephalitis, neuroborreliosis or systemic lupus erythematosus with central nervous system involvement indicating disturbances of the BBB $[16,17]$. In this context, our data give evidence to an involvement of the vascular system and chronic immune activation in the pathophysiology of mood disorders. Elevation or pathological expression of sICAM-1 might, independent of mood state, represent a mechanism for a subclinical BBB dysfunction which en- ables pro-inflammatory cytokines to enter the brain and induce depressive symptoms. Baldwin and O'Brien [18] emphasized a concept of 'vascular depression' in which changes in the vascular integrity might be involved in metabolic changes in the central nervous system, possibly followed by neurotransmitter or neurotoxic and structural changes. Increased expression in the brain of ICAM1 and elevated plasma levels of sICAM-1 have been reported in patients with depression after acute coronary syndrome and in elderly patients $[8,10,19]$. Data from van Sloten et al. [19] further supported the view that endothelial dysfunction might be associated with the pathobiology of depression in elderly people. For patients with recent acute coronary syndromes, Lesperance et al. [8] could demonstrate an association between depression and elevated sICAM-1 levels even after adjustment for potential confounders (gender, smoking, presence of metabolic syndrome). sICAM-1 was also shown to predict depression symptom severity after 12 months in heart failure patients [20]. Our trial with elevations in sICAM-1 in a group of younger patients with mood disorders might indicate that activation of cellular adhesion molecules is not limited to elderly people or patients with cardiovascular problems.

A very recent trial found increased ICAM and vascular cell adhesion molecule levels in bipolar patients during their first manic episode. The authors also found a weak correlation between ICAM levels and the severity of manic symptoms measured by the YMRS [21]. In contrast to our data, cell adhesion molecules decreased during subsequent remission and did not differ when compared to healthy controls. However, data are limited to mania, while patients with depression were excluded. In addition to its possible role as a vascular risk factor during inflammatory conditions, sICAM-1 was shown to be increased in patients with adiposity, metabolic syndrome, diabetes and dyslipidemia, and in smokers as a common risk factors for vascular disorders [21-23]. However, in the trial by Turan et al. [21], the increase in cellular adhesion molecules was independent of all other cardiovascular risk factors besides smoking behavior. People with unipolar or bipolar affective disorders are more prone to develop diabetes mellitus and vascular disease. On the other hand, adiposity and diabetes are associated with an increased risk for depressive mood changes, possibly associated with chronic low-grade inflammation [24, 25]. In this context, sICAM-1 elevation might be a common pathogenetic factor for mood disorders and mood changes during metabolic disorders, diabetes, chronic low-grade inflammation as well as vascular complications, and would
Schaefer/Sarkar/Schwarz/Friebe 
also support the hypothesis of a subclinical increased permeability of the BBB [26]. Future trials may also assess the relationships adhesion molecule expression with steroid regulation. Finally, functional changes in BBB permeability might have a significant influence on the clinical efficacy and tolerability of medication, which has to be investigated in larger trials.

\section{Limitations}

Our data are limited by the relatively low number of patients per group and the lack of evaluation of other possible relevant factors for sICAM levels such as adiposity, diabetes, dyslipidemia, smoking behavior, hypertension or leptin levels. The inclusion criterion of a period of 4 weeks in a stable state for euthymic patients might be too short. We further cannot conclude from our data that the increase in sICAM-1 levels is specific for patients with mood disorders. Differences in sICAM-1 levels between patients and controls may also be related to pharmacological treatment. However, a direct influence of different antidepressants on sICAM-1 levels was not detected, neither in a recent mouse model [14] nor in a clinical trial [13]. Finally, a direct correlation of sICAM-1 concentrations and $\mathrm{BBB}$ dysfunction has so far not been shown.

Taken together, our data offer the first evidence for a basal elevation of sICAM-1 in patients with unipolar and bipolar affective disorders, indicating chronic subclinical activation of the immune system and endothelial activation and dysfunction in the BBB.

\section{Acknowledgements}

This work was supported by Stanley Medical Research Institute Grant \#01-05.7.

\section{References}

1 Munkholm K, Brauner JV, Kessing LV, Vinberg M: Cytokines in bipolar disorder vs healthy control subjects: a systematic review and meta-analysis. J Psychiatr Res 2013;47: 1119-1133.

2 Munkholm K, Vinberg M, Vedel KL: Cytokines in bipolar disorder: a systematic review and meta-analysis. J Affect Disord 2013;144: 16-27.

3 Dowlati Y, Herrmann N, Swardfager W, Liu H, Sham L, Reim EK, et al: A meta-analysis of cytokines in major depression. Biol Psychiatry 2010;67:446-457.

4 Baraczka K, Nekam K, Pozsonyi T, Jakab L, Szongoth M, Sesztak M: Concentration of soluble adhesion molecules (sVCAM-1, sICAM-1 and sL-selectin) in the cerebrospinal fluid and serum of patients with multiple sclerosis and systemic lupus erythematosus with central nervous involvement. Neuroimmunomodulation 2001;9:49-54.

5 Kraus J, Oschmann P, Engelhardt B, Schiel C, Hornig C, Bauer R, et al: Soluble and cell surface ICAM-1 as markers for disease activity in multiple sclerosis. Acta Neurol Scand 1998; 98:102-109.

6 Rieckmann P, Nunke K, Burchhardt M, Albrecht M, Wiltfang J, Ulrich $M$, et al: Soluble intercellular adhesion molecule- 1 in cerebrospinal fluid: an indicator for the inflammatory impairment of the blood-cerebrospinal fluid barrier. J Neuroimmunol 1993;47:133-140.

7 Dimopoulos N, Piperi C, Salonicioti A, Mitsonis C, Liappas I, Lea RW, et al: Elevation of plasma concentration of adhesion molecules in late-life depression. Int J Geriatr Psychiatry 2006;21:965-971.
8 Lesperance F, Frasure-Smith N, Theroux P, Irwin $\mathrm{M}$ : The association between major depression and levels of soluble intercellular adhesion molecule 1, interleukin-6, and C-reactive protein in patients with recent acute coronary syndromes. Am J Psychiatry 2004;161: 271-277.

9 Schaefer M, Horn M, Schmidt F, SchmidWendtner MH, Volkenandt M, Ackenheil M, et al: Correlation between sICAM-1 and depressive symptoms during adjuvant treatment of melanoma with interferon-alpha. Brain Behav Immun 2004; 18:555-562.

10 Thomas AJ, Ferrier IN, Kalaria RN, Woodward SA, Ballard C, Oakley A, et al: Elevation in late-life depression of intercellular adhesion molecule- 1 expression in the dorsolateral prefrontal cortex. Am J Psychiatry 2000; 157:1682-1684

11 Jain S, Mills PJ, von Kanel R, Hong S, Dimsdale JE: Effects of perceived stress and uplifts on inflammation and coagulability. Psychophysiology 2007;44:154-160.

12 Juengst SB, Kumar RG, Failla MD, Goyal A, Wagner AK: Acute inflammatory biomarker profiles predict depression risk following moderate to severe traumatic brain injury. J Head Trauma Rehabil 2015;30:207-218.

13 van Zyl LT, Lesperance F, Frasure-Smith N, Malinin AI, Atar D, Laliberte MA, et al: Platelet and endothelial activity in comorbid major depression and coronary artery disease patients treated with citalopram: the Canadian Cardiac Randomized Evaluation of Antidepressant and Psychotherapy Efficacy Trial (CREATE) biomarker sub-study. J Thromb Thrombolysis 2009;27:48-56.
14 Isingrini E, Belzung C, d'Audiffret A, Camus $\mathrm{V}$ : Early and late-onset effect of chronic stress on vascular function in mice: a possible model of the impact of depression on vascular disease in aging. Am J Geriatr Psychiatry 2011; 19:335-346.

15 Page AV, Liles WC: Biomarkers of endothelial activation/dysfunction in infectious diseases. Virulence 2013;4:507-516.

16 Moniuszko A, Pancewicz S, Czupryna P, Grygorczuk S, Swierzbinska R, Kondrusik M, et al: ssICAM-1, IL-21 and IL-23 in patients with tick borne encephalitis and neuroborreliosis. Cytokine 2012;60:468-472.

17 Stielke S, Keilhoff G, Kirches E, Mertens PR, Neumann KH, Tsokos GC, et al: Adhesion molecule expression precedes brain damages of lupus-prone mice and correlates with kidney pathology. J Neuroimmunol 2012;252: 24-32.

18 Baldwin RC, O’Brien J: Vascular basis of lateonset depressive disorder. Br J Psychiatry 2002;180:157-160.

19 van Sloten TT, Schram MT, Adriaanse MC, Dekker JM, Nijpels G, Teerlink T, et al: Endothelial dysfunction is associated with a greater depressive symptom score in a general elderly population: the Hoorn Study. Psychol Med 2014;44:1403-1416.

20 Wirtz PH, Redwine LS, Linke S, Hong S, Rutledge T, Greenberg BH, et al: Circulating levels of soluble intercellular adhesion molecule-1 (sICAM-1) independently predict depressive symptom severity after 12 months in heart failure patients. Brain Behav Immun 2010;24:366-369. 
21 Turan C, Kesebir S, Süner O: Are ICAM, VCAM and E-selectin levels different in first manic episode and subsequent remission? J Affect Disord 2014;163:76-80.

22 Abe Y, El Masri B, Kimball KT, Pownall H, Reilly CF, Osmundsen K, et al: Soluble cell adhesion molecules in hypertriglyceridemia and potential significance on monocyte adhesion. Arterioscler Thromb Vasc Biol 1998;18:723731.
23 Demerath E, Towne B, Blangero J, Siervogel RM: The relationship of soluble ICAM-1, VCAM-1, P-selectin and E-selectin to cardiovascular disease risk factors in healthy men and women. Ann Hum Biol 2001;28:664-678.

24 Capuron L, Poitou C, Machaux-Tholliez D, Frochot V, Bouillot JL, Basdevant A, et al: Relationship between adiposity, emotional status and eating behaviour in obese women: role of inflammation. Psychol Med 2011;41: $1517-1528$
25 Fiore V, Marci M, Poggi A, Giagulli VA, Licchelli B, Iacoviello M, et al: The association between diabetes and depression: a very disabling condition. Endocrine 2015;48:14-24.

26 Hamdani N, Doukhan R, Kurtlucan O, Tamouza R, Leboyer M: Immunity, inflammation, and bipolar disorder: diagnostic and therapeutic implications. Curr Psychiatry Rep 2013;15:387. 